Title: Dual-Scattering Near-Field Microscope for Correlative Nanoimaging of SERS and Electromagnetic Hotspots

\author{
Author(s): Kusch, P., Mastel, S., Mueller, N. S., Morquillas Azpiazu, N., Heeg, S., Gorbachev, \\ R., ... Hillenbrand, R.
}

Document type: Postprint

Terms of Use: $\quad$ Copyright applies. A non-exclusive, non-transferable and limited right to use is granted. This document is intended solely for personal, non-commercial use.

Citation:

Kusch, P., Mastel, S., Mueller, N. S., Morquillas Azpiazu, N., Heeg, S., Gorbachev, R., ... Hillenbrand, R. (2017). Dual-Scattering Near-Field Microscope for Correlative Nanoimaging of SERS and Electromagnetic Hotspots. Nano Letters, 17(4), 2667-2673. https://doi.org/10.1021/acs.nanolett.7b00503 This document is the Accepted Manuscript version of a Published Work that appeared in final form in Nano Letters, copyright $\odot$ American Chemical Society after peer review and technical editing by the publisher. To access the final edited and published work see http://dx.doi.org/10.1021/acs.nanolett.7b00503. 


\section{Dual-scattering near-field microscope for correlative nanoimaging of SERS and electromagnetic hotspots}

Patryk Kusch ${ }^{1,2}$, Stefan Mastel ${ }^{2}$, Niclas S. Mueller ${ }^{1}$, Nieves Morquillas Azpiazu², Sebastian Heeg $^{3}$, Roman Gorbachev ${ }^{4}$, Fredrik Schedin ${ }^{4}$, Uwe Hübner ${ }^{5}$, Jose I. Pascual ${ }^{2,6}$, Stephanie Reich ${ }^{1}$ and Rainer Hillenbrand ${ }^{6,7^{*}}$

${ }^{1}$ Freie Universität Berlin, 14195 Berlin, Germany

${ }^{2}$ CIC nanoGUNE, 20018 Donostia, Spain

${ }^{3}$ Photonics Laboratory, ETH Zürich, 8093 Zürich, Switzerland

${ }^{4}$ The School of Physics \& Astronomy, The University of Manchester, Manchester M13 9PL, United Kingdom

${ }^{5}$ Leibniz Institute of Photonic Technology, 07745 Jena, Germany

${ }^{6} I K E R B A S Q U E$, Basque Foundation for Science, 48011 Bilbao, Spain

${ }^{7}$ CIC nanoGUNE and UPV/EHU, 20018 Donostia, Spain

*r.hillenbrand@,nanogune.eu

KEYWORDS SERS, scattering-type SNOM, Raman scattering, plasmon, hotspot mapping

\section{ABSTRACT}

Surface-enhanced Raman spectroscopy (SERS) enables sensitive chemical studies and materials identification, relying on electromagnetic (EM) and chemical-enhancement mechanisms. Here we introduce a tool for the correlative nanoimaging of EM and SERS hotspots - areas of strongly enhanced EM fields and Raman scattering, respectively. To that end, we implemented a grating spectrometer into a scattering-type scanning near-field optical microscope (s-SNOM) for mapping of both the elastically and inelastically (Raman) scattered light from the near-field probe - a sharp silicon tip. With plasmon-resonant gold dimers - canonical SERS substrates - we demonstrate with nanoscale spatial resolution that the enhanced Raman scattering is strongly correlated with enhanced elastic scattering, the latter providing access to the EM-field enhancement at the illumination frequency. Our technique has wide application potential, in the correlative nanoimaging of local-field enhancement and SERS efficiency as well as in the investigation and quality control of novel SERS substrates. 
Surface-enhanced Raman scattering (SERS) spectroscopy is a highly sensitive method to obtain vibrational information - and thus chemical information - of materials placed on rough metal surfaces $^{1-3}$, metal nanoparticles ${ }^{4-6}$ and rationally-designed optical antennas ${ }^{7}$. It has thus wide application potential in biochemical sensing ${ }^{8,9}$, analysis of art work ${ }^{10,11}$ and even single molecule studies ${ }^{5,12-15},{ }^{16-20}$. Compared to conventional Raman spectroscopy, in SERS the Raman signals are increased by many orders of magnitude $\left(10^{8}-10^{10}\right)^{21-23}$.

The enhancement of the Raman scattering originates mainly from highly concentrated (enhanced and confined) electromagnetic fields (EM hotspots) caused by plasmon excitation in metal nanostructures 1,2,12 22, 24, 25. EM hotspots can be as small as a few nanometers, while their field enhancement depends on the morphology and chemical composition of the nanostructure ${ }^{12422} 21$, 26-28. By changing the geometry of the nanostructures, one can tailor and engineer the field enhancement and, ultimately, the SERS intensities ${ }^{21,29}$. Further, a chemical enhancement may arise from the interaction between molecular and metal electron wave functions ${ }^{28}$. Both enhancement mechanisms occur on the nanometer-length scale and thus make SERS a highlylocalized phenomenon. While the enhancements of the local EM fields and Raman signals (EM and SERS enhancements, respectively) can be mapped by various methods individually (see below), a tool for the correlative nanoimaging of both quantities has not been reported yet ${ }^{29-33}$.

Nanoscale mapping of the EM-field distributions and EM hotspots can be achieved by near-field optical microscopy $(\mathrm{SNOM})^{34-39}$, photoemission electron microscopy $(\mathrm{PEEM})^{40}$, ${ }^{41}$, electron energy loss spectroscopy (EELS) ${ }^{42}$, cathode luminescence imaging ${ }^{43}$ or photo-induced force mapping ${ }^{44}$. While all of these techniques map the EM hotspots at the excitation frequency, SERS benefits from the field enhancement at both the excitation and the Raman frequency. Therefore, mapping the EM-field enhancement at a single laser (excitation) frequency does not provide comprehensive information on SERS efficiency and distribution.

Alternatively, single-molecule fluorescence and Raman imaging ${ }^{45-48}$ can be used to directly visualize the enhanced signal of interest - fluorescence or Raman scattering - but do not provide information about the field enhancement at the excitation frequency. A scanning-probe technique was recently proposed for nanoscale mapping of the SERS from EM hotspots ${ }^{40}$. A gold probe was functionalized with Raman-active molecules and scanned over a SERS substrate, while measuring SERS as a function of tip position. Although nanoscale SERS images were obtained, the technique 
does not provide access to the EM field distribution at the illumination frequency. Moreover, gold tips act as optical antennas and may modify or even dominate the SERS images due to tip-substrate coupling. ${ }^{49}$.

Here, we introduce dual-scattering scanning near-field optical microscopy (dual s-SNOM) for mapping hotspots at the frequencies of both the incoming laser radiation and the Raman-scattered light. It records the elastically (Rayleigh) and inelastically (Raman) scattered light from one and the same scanning-probe tip: in this work we use a standard silicon atomic-force microscope (AFM) tip. Silicon tips are strong Raman scatterers and suitable for elastic near-field scattering with little or no distortion of the samples' near-field distribution ${ }^{49-51}$. AFM silicon tips are readily available with sharp and ultrasharp tip diameter (10 and $2 \mathrm{~nm}$, respectively, see methods) to probe sub-10 $\mathrm{nm}$ narrow gaps between nanoparticle assemblies. We realize dual s-SNOM by implementing a Raman spectrometer into a scattering-type scanning near-field optical microscope (s-SNOM), and verify it by imaging well-defined plasmon-resonant gold dimers ${ }^{6,44,52}$. Standard s-SNOM operation maps the plasmon-enhanced elastic light scattering ${ }^{34,37}$, whereas the recording of the Raman scattering from the silicon tip yields nanoscale-resolved SERS images. Finally, we correlate the enhancement of elastically and Raman scattered light and find its spatial distribution to be in excellent agreement with numerical finite-difference time-domain (FDTD) EM enhancement generated by the gold dimers.

The EM-enhancement mechanism of surface-enhanced elastic and Raman scattering is shown in Fig. 1(a), where we illustrate both the elastic and Raman scattering from an object (O) near a plasmon-resonant structure. As the object we consider a deeply subwavelength-scale nanoparticle in the gap of a gold dimer. The incoming field $E_{\text {in }}$ of frequency $\omega_{0}$ is enhanced by a factor $f_{0}$, owing to plasmon excitation in the gold dimer. Thus, the object is illuminated by the enhanced local field $E_{l o c} \propto f_{0} E_{i n}$. The gold dimer also enhances the light scattering off the object. For inelastic scattering at the Raman-shifted frequency $\omega_{R}$, where we denote the (frequency dependent) enhancement factor by $f_{R}$, the total field amplitude enhancement after inelastic scattering is given by $f_{R} f_{0}^{21,23,50}$. If the difference $\Delta \omega$ between $\omega_{R}$ and $\omega_{0}$ is small compared to the spectral width of the plasmon resonance, we can approximate the enhancement of the scattered Raman field amplitude by $f_{0}{ }^{2}$ according to $f_{0} \approx f_{R}$. For elastic light scattering $\left(\omega_{0}=\omega_{R}\right)$, where the enhancement factors are identical $\left(f_{0}=f_{R}\right)$, we obtain a field amplitude enhancement $f_{0}^{221,50}$ 
(note that for this consideration the illumination and detection direction have to be the same, which is a consequence of the reciprocity theorem).

(a)

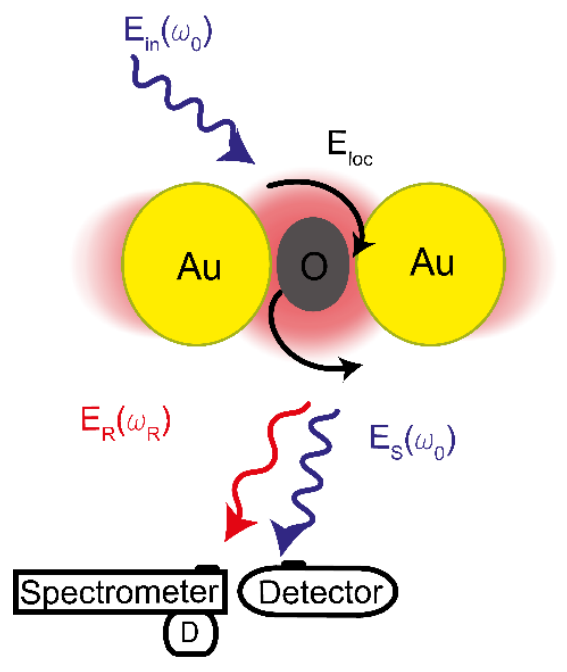

(b)

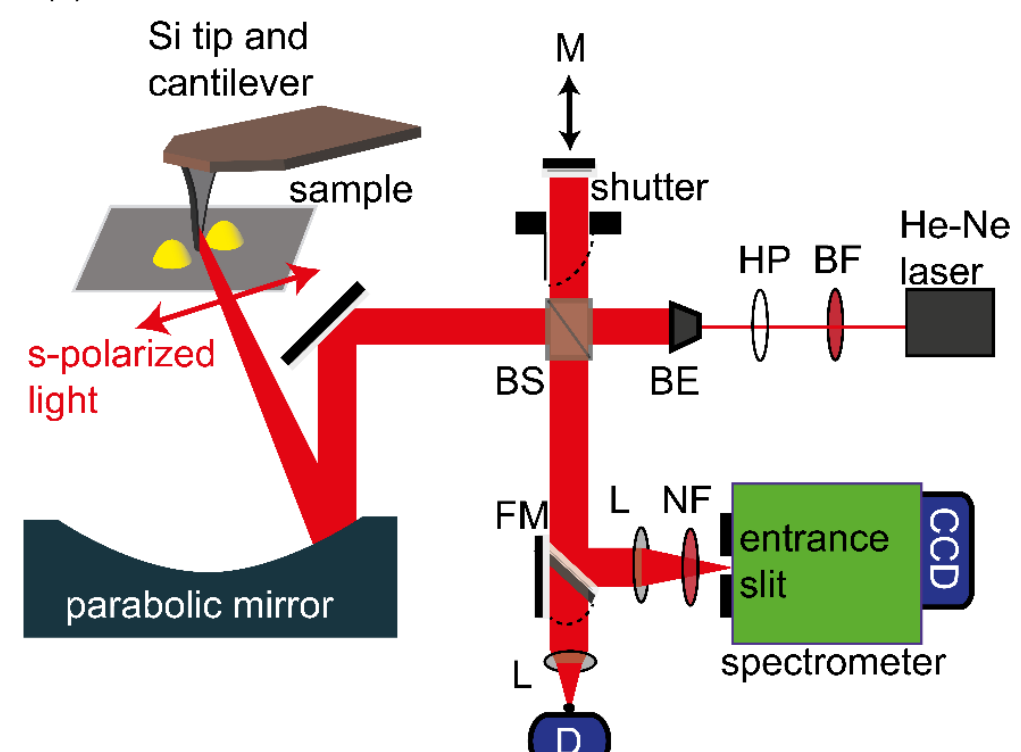

Figure 1 Principle and setup of dual s-SNOM. a) Elastic and Raman-scattered light from an object $\mathrm{O}$ in the presence of a plasmonic dimer. $E_{\text {in }}$ denotes the incoming field, which is converted into an enhanced and localized EM field $E_{l o c}$ by the dimer that interacts with the object. $E_{R}$ (Raman-scattered) and $E_{S}$ (elastically scattered) are the fields radiated by the object via the dimer. b) s-SNOM and Raman setup employing a Si tip as scattering probe. A He-Ne laser is collimated with a beam expander (BE) and focused with a parabolic mirror onto the tip apex, after passing a bandpass filter (BF) for cutting out plasma lines in the emission spectrum of the laser. To choose between s- and p-polarized light, a half-wave plate (HP) is installed before the (BE). The elastically backscattered field is measured with a pseudo-heterodyne Michelson interferometer comprising a beam splitter (BS), a vibrating mirror (M) and a detector (D). Raman spectra are recorded by directing the backscattered light via a flip mirror (FM) and notch filter (NF) into a grating spectrometer.

Consequently, the increase of the intensity of the elastically and Raman-scattered light scales with the fourth power of the local field enhancement, $I_{S, R} \propto f^{4}$. Correlating elastically and Ramanscattered intensity from a nanoscale object opens the possibiltiy to experimentally explore differences between the elastic and inelastic scattering events (potentially caused due to $\omega_{0} \neq \omega_{R}$ and additional chemicial-enhancement effects in SERS), to study the efficiency of SERS substrates, and to test novel theories of SERS. 
We implemented dual-scattering near-field microscopy [based on the principle shown in Fig. 1(a)] with a commercial s-SNOM (NeaSNOM from Neaspec GmbH, Germany). A conventional scattering near-field probe (Si tip) mimics the scattering object. For nanoimaging the plasmonic hotspots we scan the tip across the SERS substrate and record the elastically and Raman-scattered light. Specifically, we use ultra-sharp (Nanosensors SSS-NCHR; tip diameter $2 \mathrm{~nm}$ ) and sharp (Nanosensors ARROW NCR; tip diameter $10 \mathrm{~nm}$ ) commercial AFM tips. A parabolic mirror is used (i) to focus s-polarized (perpendicular to tip, i.e. parallel to the surface of the SERS substrate) light of a He-Ne laser (633 nm wavelength) onto the scanning-probe tip and (ii) to collect the backscattered field. As typical for s-SNOM, the tip oscillates vertically at the cantilever's mechanical resonance frequency $\Omega(300 \mathrm{kHz})$. For mapping the $s$-polarized elastically-scattered field from the tip, we employ a Michelson interferometer. By demodulating the detector signal at a higher harmonic of the tapping frequency, $n \Omega$, background contributions are suppressed ${ }^{53}$. Applying a pseudo-heterodyne interferometric detection scheme, where the reference mirror oscillates at a frequency $M \ll \Omega$, we record amplitude $\left|E_{n}\right|$ and phase $\phi_{n}$ images of the elastically scattered light simultaneously to topography (conventional s-SNOM operation). To detect Raman scattered light from the tip, we implemented a single-grating spectrometer (Acton SP2500i, Princeton instruments) with a silicon charge-coupled device (CCD) for recording the spectra (iXON ultra, Andor). A flip mirror [FM in Fig. 1(b)] is used to direct the tip-scattered light to the Raman spectrometer. A notch filter (NF) in front of the spectrometer suppresses elasticallyscattered light, as its intensity is more than $10^{6}$ times larger than that of the Raman-scattered intensity.

We applied the dual-scattering near-field microscope to image well-defined gold nanodimers ${ }^{6,44}$, 52 , which represent an efficient SERS substrate. They consist of two closely spaced gold nanoparticles with spherical shape [linescan Fig. 2 (a), (d), (g)] of about $100 \mathrm{~nm}$ diameter and 70 $\mathrm{nm}$ height on a $300 \mathrm{~nm}$ thick $\mathrm{SiO}_{2}$ layer on a Si substrate, fabricated by e-beam lithography. The distance between the nanoparticles varies from $35 \mathrm{~nm}$ to $72 \mathrm{~nm}$ (the exact gap sizes were determined by scanning electron microscopy), while the plasmon resonance is close to $633 \mathrm{~nm}^{6}$, 52. To visualize the dimers, we first recorded topography images [Fig. 2 (b), (e) and (h)] using an ultrasharp tip. Using the same tip, now serving as nanoscale Raman scatterer, we subsequently recorded Raman spectra [Fig. 2 (c), (f) and (i)] when the tip was positioned either exactly inside the gap [illustrated in Fig. 2 (a), (d) and (f)] or about $100 \mathrm{~nm}$ away from the dimer. Both inside 
and outside the gap we observed a strong silicon Raman peak at $\Delta \omega=521 \mathrm{~cm}^{-1}$ (longitudinal optical phonon, corresponding to a shift of $65 \mathrm{meV}$ ).
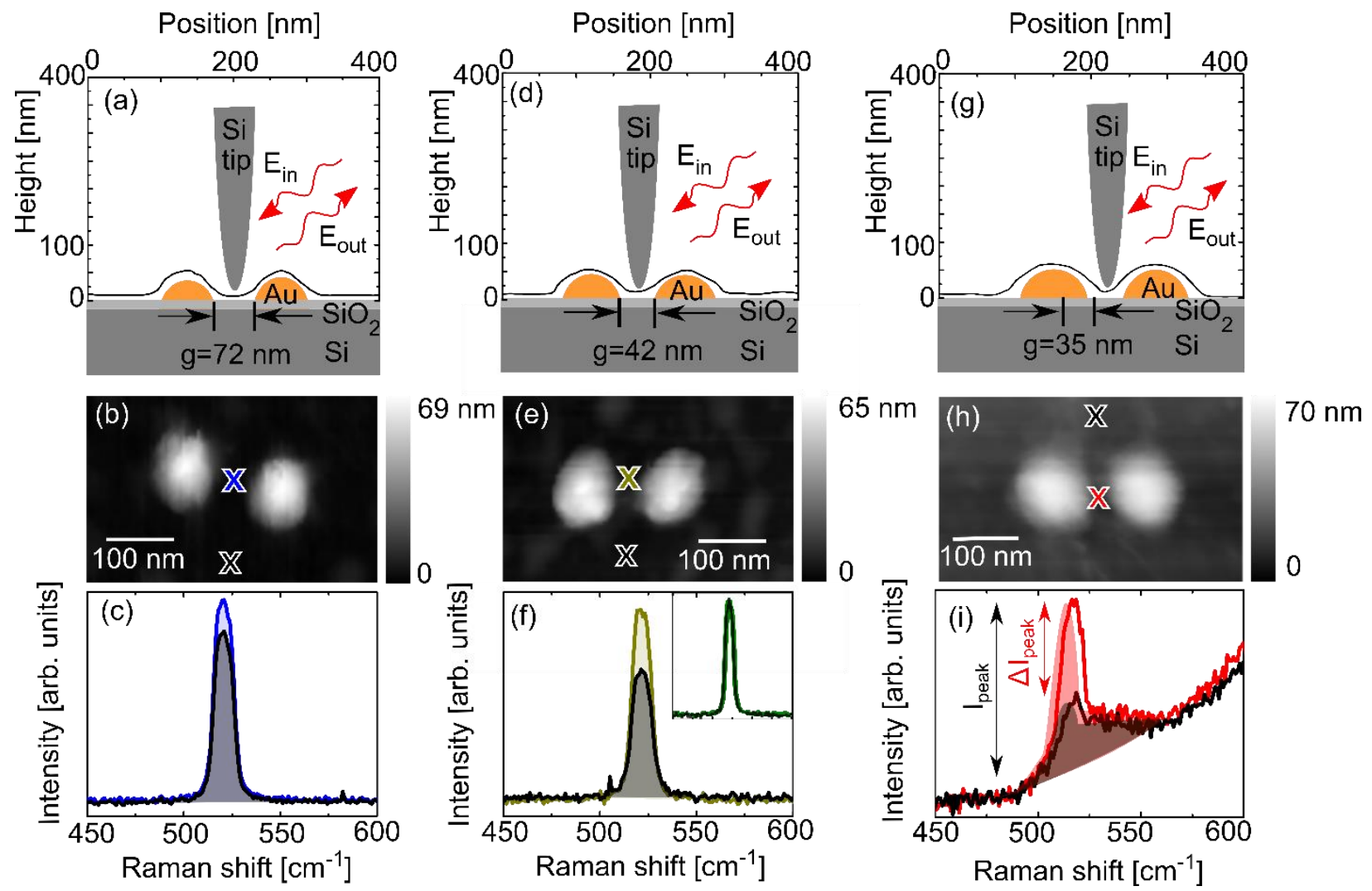

Figure 2 SERS form an ultrasharp Si tip in plasmonic hotspots. (a), (d), (g) Schematics of the experiment. (b), (e), (h) Topography images of gold dimers with gap width $g=72 \mathrm{~nm}, g=42 \mathrm{~nm}$ and $g=35 \mathrm{~nm}$. (c), (f), (i) Raman spectra of Si tip inside (blue, green, red) and outside (black) the gap. The strong background for the dimer with $\mathrm{g}=35 \mathrm{~nm}$ in (i) is explained by the strong nearfield enhancement yielding a strong photoluminescence of gold nanostructures ${ }^{54}$. Note: inset in (f) show Raman spectra when the tip is inside (green) and outside (black) the gap for s-plarized light perpendicular to dimer axis.

Interestingly, the peak intensity was considerably increased by $\Delta I_{\text {peak }}$ when the tip is inside the gap [Fig. 2 (c) (f) and (i); note that $I_{\text {peak }}$ denote the area under the peak]. This finding clearly demonstrates SERS from the tip apex, which originates from the strongly-enhanced local EM field, $E_{l o c}$, inside the gap, despite the relatively large gap widths $g$. The SERS signal $\Delta I_{\text {peak }}$ increased with decreasing $g$, owing to the increasing gap fields that originate from the increasing plasmonic coupling of the two nanoparticles ${ }^{29,55-57}$. For the dimer with $g=35 \mathrm{~nm}$, the increase of the total Raman-scattering intensity, $\Delta I_{\text {peak }} / I_{\text {peak }}=86 \%$, is remarkably strong, considering that only the 
very tip apex couples to the nanodimer hotspot (for $g=72 \mathrm{~nm}$ and $g=42 \mathrm{~nm}$ we found $\Delta I_{\text {peak }} / I_{\text {peak }}=16 \%$ and $\left.\Delta I_{\text {peak }} / I_{\text {peak }}=59 \%\right)$. To verify that the strong field in the dimer gap originates from plasmon coupling between the gold nanoparticles, we rotated the dimer with $g=$ $42 \mathrm{~nm}$ gap size by $90^{\circ}$ and recorded Raman spectra when the tip is located inside and outside the gap. In this configuration, the s-polarized light is oriented perpendicular to the dimer axis. The spectra [inset Fig. 2 (f)] clearly show that the Raman spectrum and particularly the peak intensities are identical, revealing that the field enhancement inside the gap - as expected ${ }^{6}$ - is negligible small. This shows that the SERS signal from a silicon tip is an excellent means for characterizing plasmonic nanostructures. To estimate the local-field and SERS enhancement, we calculated the tip volume illuminated by the diffraction-limited laser focus, $V_{L S}$, and by the hotspot in the dimer gap, $V_{H S}$. We assumed that the tip is a cone of $1 \mathrm{~nm}$ apex radius and 32 degree apex angle. Considering that the laser focus illuminates the very cone tip on a length of $1 \mu \mathrm{m}$, we found that the Raman intensity measured on the substrate [black curves in Fig. 2 (c), (f), (i)] originates from a Si volume of about $V_{L S}=0.088 \mu \mathrm{m}^{3}$. The increase $\Delta I_{\text {peak }}$ is caused by SERS of the tip volume illuminated by the plasmonic hot spot in the gap. Assuming a homogeneous illumination of the very cone tip on a length of $70 \mathrm{~nm}$ (corresponding to the height of the $g=35 \mathrm{~nm}$ dimer), we obtain $V_{H S}=3.4 \cdot 10^{-5} \mu m^{3}$. For the Raman enhancement factor we subsequently obtain $\mathrm{F}=$ $\left(\Delta I_{\text {peak }} / I_{\text {peak }}\right) /\left(V_{H S} / V_{L S}\right)=2200$. It corresponds to a field enhancement factor $f=F^{1 / 4}=6.8$, which agrees with the numerically-calculated field enhancement in $f=\sqrt{f_{x}^{2}+f_{z}^{2}}$ inside the dimer gap [see methods].

To visualize the spatial distribution of the SERS hotspots, we scanned the tip through the dimer gaps, perpendicular to the dimer axes, as illustrated in the inset of Fig. 3 and along the dimer axis. At each tip position $y$ we recorded a SERS spectrum and determined $\Delta I_{\text {peak }}(y)$ (Fig. 3) by subtracting the Raman spectrum obtained on the substrate. We observe a clear maximum of $\Delta I_{\text {peak }}(y)$ inside the gaps, where the field enhancement is strongest. The maximum increases with decreasing gap width $g$, confirming the results obtained from the single point spectra shown in Fig. 2. On the other hand, the full width of half-maximum of the line profile $\Delta I_{\text {peak }}(y)$ increases for the dimer with $35 \mathrm{~nm}$ gap. We explain this unexpected observation by the shape of the particles not being perfectly spherical. Indeed, the $35 \mathrm{~nm}$ wide gap is more elongated than that of the dimers 
with larger gap widths [compare topography images Figs. 2 (b),(e),(h)], yielding a broader nearfield distribution perpendicular to the gap.

In Fig. 4, we compare the spatial distribution of experimental SERS intensities with the numerically calculated spatial near-field distribution (FDTD calculation) for the dimer shown in Fig. 2 (d) and (e). To that end, we modeled the geometry of the gold dimer by two half ellipsoids. Its height $(60 \mathrm{~nm})$, diameter $(88 \mathrm{~nm})$ and separation (gap size, $42 \mathrm{~nm}$ ) were extracted from the topography image shown in Fig. 2(e). We note that the diameter and gap size represent good estimates, as the apex of ultrasharp tips is as small as $2 \mathrm{~nm}$.

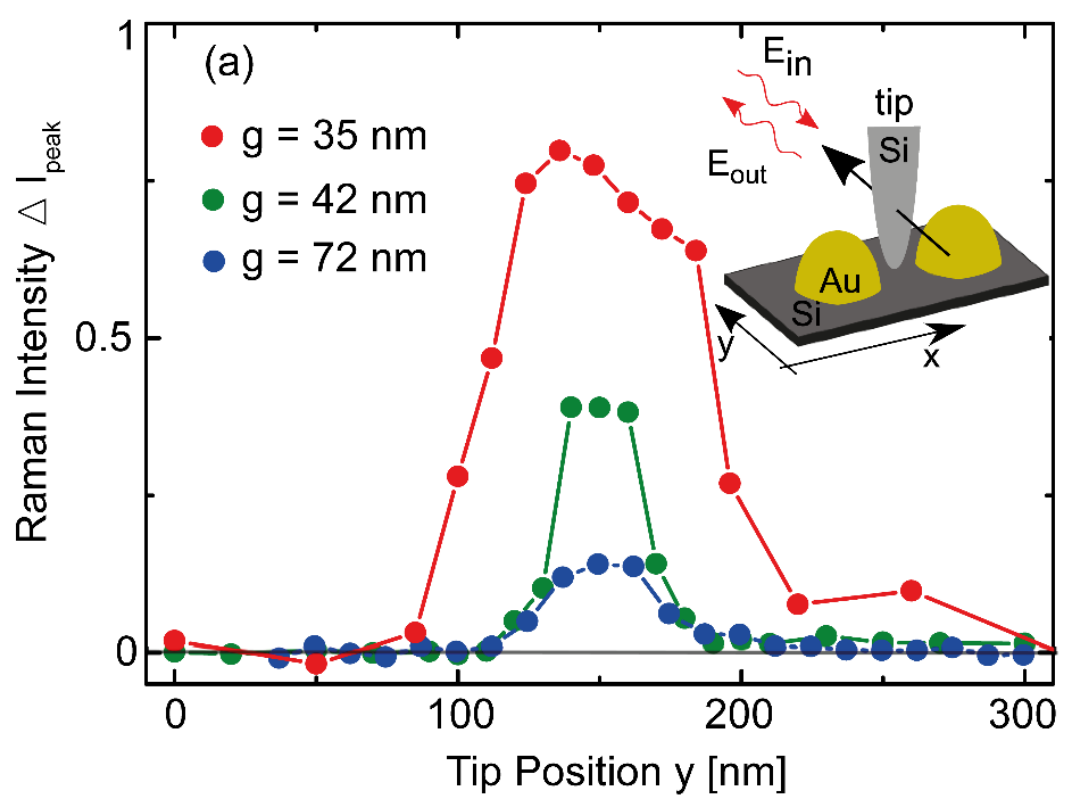

Figure 3 SERS profiles across dimer gaps obtained with an ultrasharp Si tip (same as in Fig. 2). Line profiles of the Raman intensity $\Delta I_{\text {peak }}$ through the gap of different dimers, perpendicular to the dimer axis. The scan direction is indicated by black arrow in the inset.

Figure 4(a) shows a cross section of the calculated spatial distribution of the field enhancement $f$ along the dimer axis, where $f=\sqrt{f_{x}^{2}+f_{z}^{2}}$ with $f_{x, z}$ being the field enhancement in $x$ and $z$ direction, respectively. We observe the typical strong field enhancement at the dimer extremities and inside the gap. Figure 4(b) shows a line profile of $f^{2}$ and $f^{4}$ (dashed grey and solid green curves, respectively) along the dashed white line in Fig. 4(a), which indicates the path of the tip in $10 \mathrm{~nm}$ distance to the sample surface. For comparison with our experiment, we measured $\Delta I_{\text {peak }}$ along the dimer axis. To that end, we manually positioned the ultrasharp Si tip (the same as in Fig. 2 and 
3) along the dimer axis [illustrated in the inset of Fig. 4(b)] and recorded Raman spectra. We find that $\Delta I_{\text {peak }}$ [green dots in Fig. 4(b)] exhibits a global maximum inside the gap and two weaker local maxima at the dimer extremities. The experimental SERS data match well the line profile showing the fourth power of the numerically calculated field enhancement, $f^{4}$ (green solid line). We explain the small discrepancies between the green curve and the experimental data (especially when the tip is inside the gap) by the fact that the Raman signals originate from a three-dimensional tip.
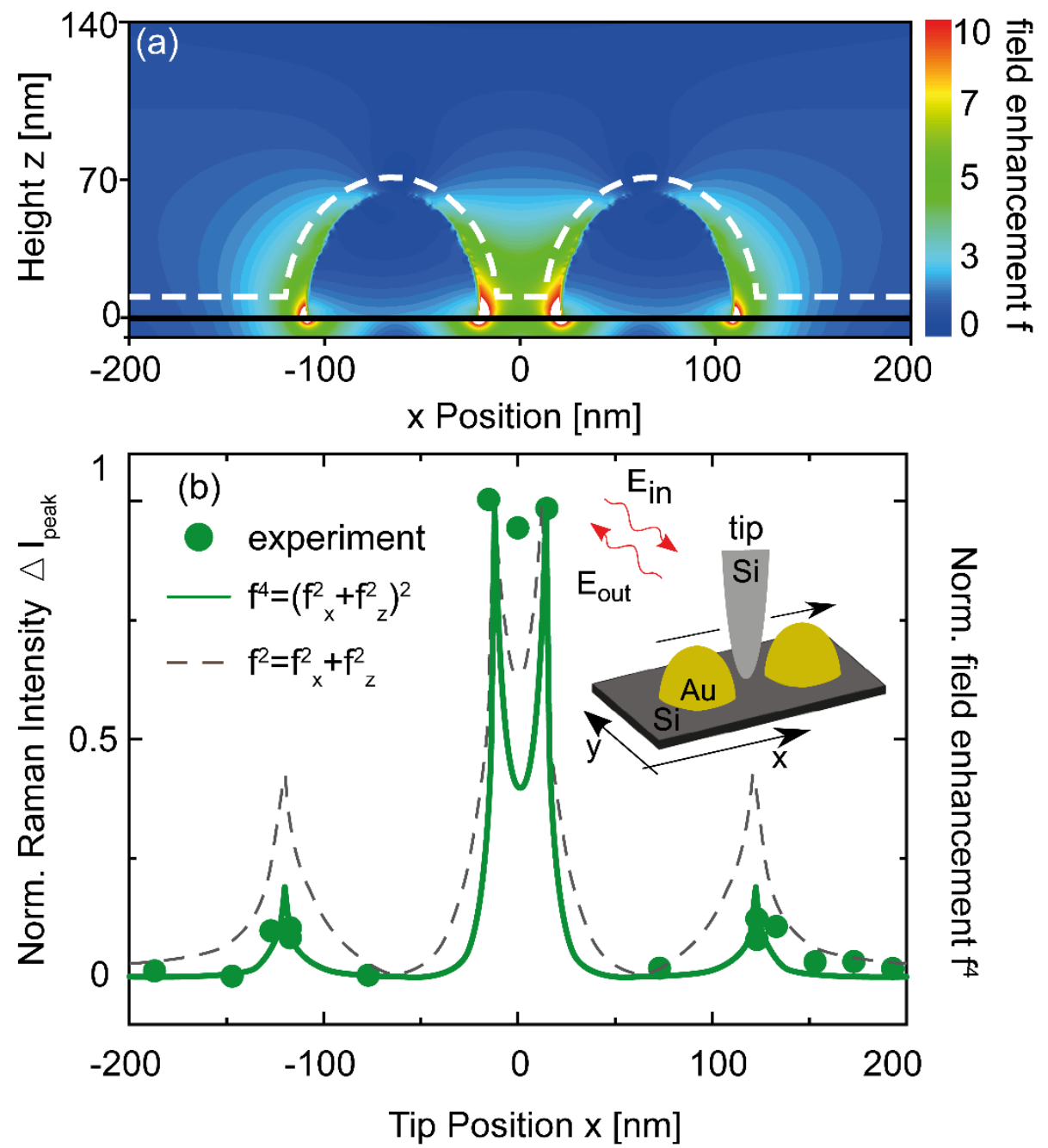

Figure 4 Comparison between calculated field enhancement and Raman intensities. (a) Cross-section of the numerically calculated spatial distribution of the field enhancement around a gold dimer with $42 \mathrm{~nm}$ gap size. (b) Normalized line profile of the Raman intensity $\Delta I_{\text {peak }}$ along the dimer axis (green dots) compared to $f^{4}$ and $f^{2}$ (normalized to the maximum Raman intensity). $f$ was extracted along the white dashed line in Fig. 4(a), which indicates the path of the tip in 10 $\mathrm{nm}$ distance to the sample surface. The Raman data were obtained with an ultrasharp tip (the same as in Fig. 2 and 3). 
The field enhancement, however, was extracted along one-dimensional path [dashed white line in Fig. 4(a)]. Further, potential drift between tip and dimer during spectra acquisition (60 s integration time per tip position) may affect the experimental Raman intensities. We corroborate the match between the SERS signal and the fourth power of the field enhancement (green solid line) by plotting the intensity enhancement profile, $f^{2}$ (grey dashed line), which exhibits a significantly worse match. The scaling of the Raman intensity with $f^{4}$ demonstrates that surface-enhanced Raman scattering from the Si tip - an individual Raman scatterer - is observed. The results further show that the SERS signal can be imaged with nanoscale spatial resolution. We explain the good agreement between the SERS signal and $f^{4}$ by the small Raman shift $\Delta \omega$ of the Si phonon (65 $\mathrm{meV}$ ) compared to the width of the plasmon resonance (typically several $100 \mathrm{meV}$ ).

The dual-scattering near-field microscope allows for a direct correlation of SERS hotspots with the EM near-field intensity. The latter is mapped by recording the elastically-scattered light from the Si tip, while recording the topography of the gold dimers, as is typically done in an s-SNOM experiment. Using a sharp Si tip (less sharp than the ultrasharp tips used in Figs. 2-4), we imaged the nanodimer with the smallest gap [ $g=35 \mathrm{~nm}$, topography shown in Fig. 5(a)], which exhibits the strongest SERS intensity [Fig. 2 (h)]. The amplitude image of the elastically-scattered field, $s_{2}$, is obtained by second-harmonic demodulation of the interferometric detector signal. It exhibits three hotspots, one inside the gap and two at the dimer extremities ${ }^{50}$. The hotspot in the gap is strongest [Fig. 5(b)], in good agreement with the numerically calculated near-field distribution [Fig. 5(d)]. We also recorded $s_{2}$ as a function of the tip-substrate distance $d$ in the gap center [Fig. 5(c)], showing that with increasing distance $d$ the amplitude signal $s_{2}$ decreases rapidly (1/e decay length of about $10 \mathrm{~nm}$ ). For $d>30 \mathrm{~nm}$, the amplitude signal $s_{2}$ reaches the noise floor, thus verifying the absence of any background signals ${ }^{58}$ and confirming the pure near-field character of the $s_{2}$ signal.

In contrast to SERS, the interferometric detection in s-SNOM probes the scattered field rather than the scattered intensity. Further, s-SNOM experiments with infrared antennas showed that the amplitude $s_{2}$ does not measure the near field (i.e. EM field enhancement $f$ ) but $f^{2}$ when $s$-polarized illumination and $s$-polarized interferometric detection is applied ${ }^{50,59}$. This finding can be explained by the double-scattering mechanism: the tip is illuminated by the plasmon-enhanced near field of 
the dimer and then elastically scatters via plasmon excitation in the dimer. In Fig. 5(e) we thus compare the experimental line profile $s_{2}$ (dots) along the dimer axis with the numerically calculated line profile $f^{2}$ (black solid line).
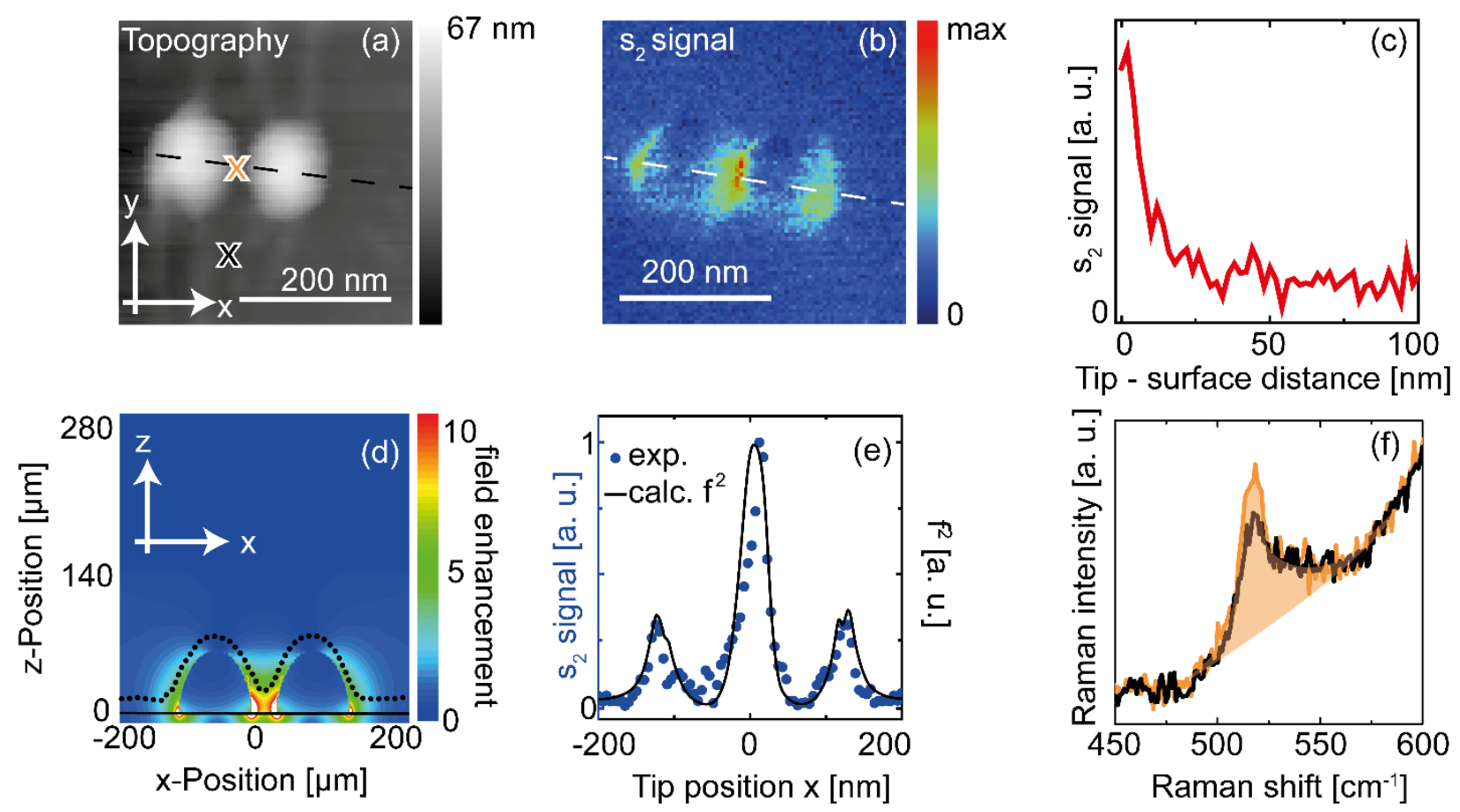

Figure 5 s-SNOM imaging of plasmonic hotspot of gold dimer with a sharp Si tips. (a) Topography image of gold dimer with gap width $g=35 \mathrm{~nm}$. The orange/black $X$ mark the tip positions for Raman experiments (b) Near-field image s2 (map of elastically scattered light), simultaneously recorded with topography. (c) s2-approach curve measured at the gap center. (d) Cross-section of the numerically calculated spatial distribution of the field enhancement around a gold dimer with $35 \mathrm{~nm}$ gap size. The dashed line shows a topography line scan extracted along the dashed line in (a). (e) Dots show the s2 line profile extracted along the dashed line in (b). Black solid line shows $f^{2}$ along the dashed line in (d). (f) Raman spectra of Si tip inside (orange) and outside (black) the gap.

For the calculation we modeled the geometry of the gold dimer by two half ellipsoids. Their height $(63 \mathrm{~nm})$ was extracted from the topography image shown in Fig. 2(e), while their diameter (100 $\mathrm{nm}$ ) and separation (gap size $\mathrm{g}=35 \mathrm{~nm}$ ) were obtained from SEM images. Figure 5(d) shows a cross section of the calculated spatial distribution of the field enhancement $f$. Similar to Fig. 4(b) we observe a strong field enhancement at the dimer extremities and inside the gap. To obtain the 
line profile $f^{2}$ along the path of the tip, we extracted $f$ along the black dashed line in Fig. 5(d) and plotted the corresponding $f^{2}$ line profile in Fig. 5(e) (black solid line). The dashed black line in Fig. 5(d) represents the experimental topography line profile extracted from Fig. 5(a), which in the numerical model was placed $10 \mathrm{~nm}$ above the sample surface [solid black line in Fig. 5(d)], in order to account for the finite tip size and vertical tip oscillation of about $20 \mathrm{~nm}$. We find an excellent agreement between the calculated profile $f^{2}$ and the near-field profile $\mathrm{s}_{2}$, showing that for $s$-polarized visible illumination and interferometric detection of the elastically backscattered $s$ polarized field our setup indeed maps a near-field amplitude $s_{2}$ that scales with the square of the field enhancement rather than with the field enhancement (while SERS scales with $f^{4}$ ). For comparing s-SNOM and SERS signals experimentally, we recorded SERS spectra of the tip inside the dimer gap [position marked by white/orange " $X$ " in Fig. 5(a)], where the elastic-scattering signal $s_{2}$ of the tip is at maximum. A second Raman spectrum was recorded at $100 \mathrm{~nm}$ distance to the gap [marked by white/black "X" in Fig. 5(a)]. As before in Fig. 2, the SERS intensity significantly increases when the tip is inside the gap [Fig. 5(f)], which correlates with the enhanced $s_{2}$ signal in the gap [Fig. 5(b)].

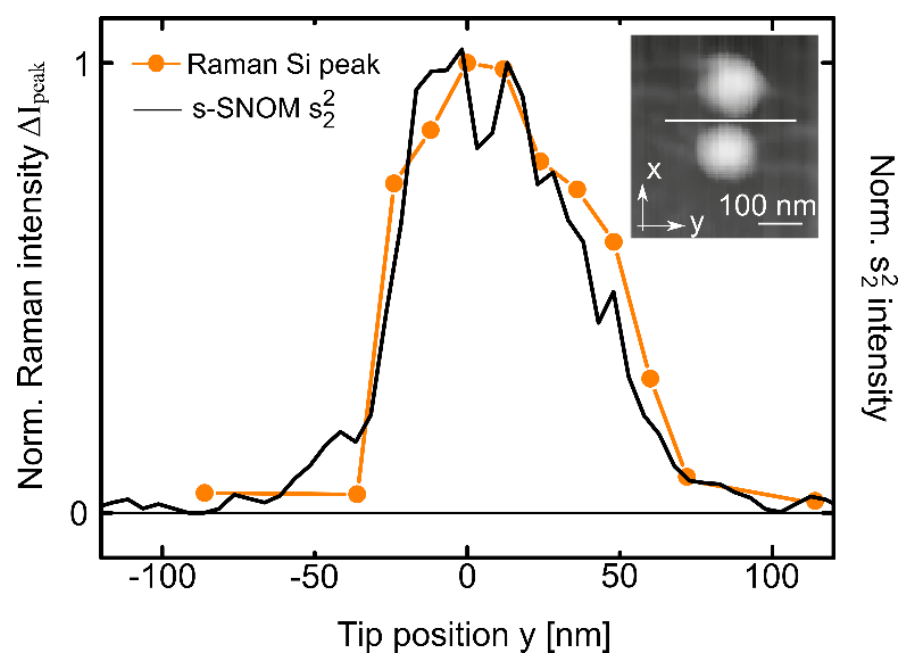

Figure 6 Comparison of s-SNOM and SERS line profiles of dimer gap obtained with a sharp Si tip. The black curve shows the s-SNOM signal s2 along the horizontal white line in the topography image (inset) of the gold dimer [extracted from Fig. 5(b)]. The orange dots show $\Delta I_{\text {peak }}$ of the Si Raman peak along the horizontal white line. Both the s-SNOM and SERS profile have been recorded with the identical Si tip. 
To compare s-SNOM and SERS profiles through the gap, we extracted the s-SNOM amplitude $s_{2}$ from Fig. 5(b) and recorded $\Delta I_{\text {peak }}$ along the horizontal white line depicted in the topography image of the dimer (inset of Fig. 6). Because $s_{2}^{2} \propto \Delta I_{\text {peak }}$ (see above), we plot the square of the sSNOM amplitude $s_{2}^{2}$ (black curve in Fig. 6) and the SERS intensity $\Delta I_{\text {peak }}$ (orange dots in Fig. 6) as a function of position. Both line profiles show the same relative dependence on position, exhibiting a maximum exactly inside the gap and a full width at half maximum (FWHM) of 75 $\mathrm{nm}$. The SERS and s-SNOM intensities in Fig. 6 demonstrate the correlation of elastic and Raman scattering for our plasmonic gold nanodimer, which in turn shows that the enhanced SERS signal is predominantly due to the EM-field enhancement. The measurements further show that for phonon energies (Raman shifts) smaller than the plasmon width the spatial distribution of the electromagnetic enhancement in SERS can be well approximated by $f^{4}$. We further conclude that chemical enhancement is negligible, as it would manifest by discrepancies between $\Delta I_{\text {peak }}$ and $s_{2}^{2}$.

We developed a dual-scattering near-field microscope based on s-SNOM for the nanoimaging for EM and SERS hotspots. It records the elastic- and Raman-scattered light from a dielectric Ramanactive probe tip scanning a (plasmonic) SERS substrate. While the maps of elastic light scattering (s-SNOM maps) show the near-field intensity distribution, the Raman maps reveal the spatial distribution of SERS. Specifically, we imaged plasmonic gold nanodimers using standard AFM silicon tips. We observed strong SERS signals inside nanodimer gaps of tens of nanometers width, which correlate well with the enhanced elastic s-SNOM signal. Nanoscale-resolved maps of SERS, squared s-SNOM signals and the fourth power of the numerically-calculated field enhancement were found to correlate well with each other. This lets us conclude that the electromagnetic/plasmonic enhancement mechanism dominates the SERS signals of silicon in the proximity of gold nanoparticles. No chemical enhancement was observed within the sensitivity of our experiment.

Dual-scattering near-field microscopy is a promising tool for screening nanoplasmonic systems for local SERS efficiency. Applying the technique to more complex plasmonic structures will reveal their EM near-field distribution together with nanoimages of their SERS activity, which will allow for quantifying EM and SERS enhancement from the same spot. We also envision to apply other probes than silicon tips, such as diamond tips (Raman shift of $160 \mathrm{meV}$ ) or silicon tips 
coated with molecules, in order to study the correlation between elastically and Raman scattered intensity for the case of larger Raman shifts and potential chemical interaction, respectively. Further, dual s-SNOM could be applied for correlative nanoimaging of elastically scattered light and photoluminescence, for example, form molecule-coated tips or from carbon nanotube tips. In the present work, mapping of elastic and Raman-scattered light was done sequentially. By synchronizing s-SNOM and Raman spectrometer, we envision the recoding of the s-SNOM, SERS and photoluminescence images simultaneously.

\section{Methods}

\section{Numerical Simulations}

The numerical simulations of the near-field distribution were conducted using the commercial finite-difference time-domain package Lumerical FDTD solutions (www.lumerical.com, Vancouver, Canada). We assume an illuminating plane wave $\boldsymbol{E}_{\text {inc }}(x, y, z)$ from above at normal incidence to the substrate with polarization parallel to the dimer axis at a free-space wavelength $\lambda=633 \mathrm{~nm}$. According to the measured topography image, the dimers were modeled as two gold half ellipsoids placed on a $3 \mathrm{~nm}$-thick Ti interlayer. The substrate was simulated as a $300 \mathrm{~nm}$-thick $\mathrm{SiO}_{2}$ layer on a silicon substrate. Light pulses of 16 fs were used to accurately simulate interference effects from the substrate.

We note that the simulations did not take into account the presence of the tip, as the plasmonic response of metal nanostructures is only weakly modified by a dielectric tip of only few nanometer apex size $\mathrm{s}^{50,60}$. Essentially, the presence of the tip leads to a small shift of the plasmon dimer resonance, which, however, is much smaller than the width of the plasmon resonance. Consequently, we can expect that any modification of the near field that is scattered by the tip and recorded as a function of tip position is negligible small. Indeed, the good agreement between the numerically calculated near-field distribution and the experimental s-SNOM and Raman images confirms the negligible influence of the tip.

\section{Porbes}

For the Raman experiments, we used two different types of silicon tips: ultra-sharp tips with an apex diameter of $2 \mathrm{~nm}$ and sharp tips with $10 \mathrm{~nm}$ apex diameter. The s-SNOM experiments were performed with sharp silicon tips, since it was not possible to measure a reliable s-SNOM signal 
from the ultra-sharp tips. This can be explained by the setup configuration and the small scattering cross section of the $2 \mathrm{~nm}$ ultra-sharp tip. Note that for elastic light scattering detection we used single line Si photodetector and demodulated the detector signal, while for Raman spectroscopy we used a highly sensitive CCD camera. Further, the integration time in s-SNOM was 30 ms per pixel, while $60 \mathrm{~s}$ integration time was used for the Raman measurements."

\section{Funding Sources}

The authors acknowledge support by the Spanish Ministry of Economy and Competitiveness (National Project MAT2015-65525-R), the Dahlem Research School DRS: Dahlem International Network PostDocs, the Deutsche Forschungsgemeinschaft within project SfB 658 and the FocusArea NanoScale for support and funding.

\section{Acknowledgements}

We thank Lukas Novotny for stimulating discussions.

\section{Competing financial interests:}

Rainer Hillenbrand is co-founder of Neaspec $\mathrm{GmbH}$, a company producing scattering-type scanning near-field optical microscope systems, such as the one used in this study. The remaining authors declare no competing financial interests.

\section{References}

1. Stiles, P. L.; Dieringer, J. A.; Shah, N. C.; Van Duyne, R. P. Annu. Rev. Anal. Chem. 2008, 1, 601-626. 2. Fleischmann, M.; Hendra, P. J.; McQuillan, A. J. Chemical Physics Letters 1974, 26, (2), 163-166.

3. Jeanmaire, D. L.; Van Duyne, R. P. Journal of Electroanalytical Chemistry and Interfacial Electrochemistry 1977, 84, (1), 1-20.

4. $\quad$ Xu, H.; Bjerneld, E. J.; Käll, M.; Börjesson, L. Physical review letters 1999, 83, 4357.

5. Kneipp, K.; Wang, Y.; Kneipp, H.; Perelman, L. T.; Itzkan, I.; Dasari, R. R.; Feld, M. S. Physical review letters 1997, 78, (9), 1667-1667.

6. Heeg, S.; Fernandez-Garcia, R.; Oikonomou, A.; Schedin, F.; Narula, R.; Maier, S. A.; Vijayaraghavan, A.; Reich, S. Nano letters 2012, 13, (1), 301-308.

7. Zhu, W.; Banaee, M. G.; Wang, D.; Chu, Y.; Crozier, K. B. Small 2011, 7, (13), 1761-1766.

8. Gao, J.; Guo, L.; Wu, J.; Feng, J.; Wang, S.; Lai, F.; Xie, J.; Tian, Z. Journal of Raman Spectroscopy 2014, 45, (8), 619-626.

9. $\quad$ Li, Q.-I.; Li, B.-w.; Wang, Y.-q. RSC Advances 2013, 3, (32), 13015-13026.

10. Sharma, B.; Frontiera, R. R.; Henry, A.-I.; Ringe, E.; Van Duyne, R. P. Materials today 2012, 15, (1), 16-25.

11. Saha, A.; Jana, N. R. ACS applied materials \& interfaces 2014, 7, (1), 996-1003. 
12. Le Ru, E. C.; Etchegoin, P. G. Annual Review of Physical Chemistry 2012, 63, 65-87.

13. Aradhya, S. V.; Venkataraman, L. Nature nanotechnology 2013, 8, (6), 399-410.

14. Nie, S.; Emory, S. R. science 1997, 275, (5303), 1102-1106.

15. Pozzi, E. A.; Zrimsek, A. B.; Lethiec, C. M.; Schatz, G. C.; Hersam, M. C.; Van Duyne, R. P. The Journal of Physical Chemistry C 2015, 119, (36), 21116-21124.

16. Le Ru, E. C.; Blackie, E.; Meyer, M.; Etchegoin, P. G. The Journal of Physical Chemistry C 2007, 111, (37), 13794-13803.

17. Laurence, T. A.; Braun, G. B.; Reich, N. O.; Moskovits, M. Nano letters 2012, 12, (6), 2912-2917.

18. Li, J. F.; Huang, Y. F.; Ding, Y.; Yang, Z. L.; Li, S. B.; Zhou, X. S.; Fan, F. R.; Zhang, W.; Zhou, Z. Y.; Ren, B.; others. nature 2010, 464, (7287), 392-395.

19. Deckert, V.; Zeisel, D.; Zenobi, R.; Vo-Dinh, T. Analytical Chemistry 1998, 70, (13), 2646-2650.

20. Rasmussen, A.; Deckert, V. Journal of Raman Spectroscopy 2006, 37, (1-3), 311-317.

21. Novotny, L.; Hecht, B., Principles of nano-optics. Cambridge university press: 2012.

22. Moskovits, M. Physical Chemistry Chemical Physics 2013, 15, (15), 5301-5311.

23. Le Ru, E.; Etchegoin, P., Principles of Surface-Enhanced Raman Spectroscopy: and related plasmonic effects. Elsevier: 2008.

24. Can $\{\backslash\{\{c\}\}$ ado, L. G.; Jorio, A.; Ismach, A.; Joselevich, E.; Hartschuh, A.; Novotny, L. Physical review letters 2009, 103, (18), 186101.

25. Xu, H.; Aizpurua, J.; K\{\"a\}II, M.; Apell, P. Physical Review E 2000, 62, (3), 4318.

26. Tay, L.-L.; Hulse, J.; Kennedy, D.; Pezacki, J. P. The Journal of Physical Chemistry C 2010, 114, (16), 7356-7363.

27. Kern, A. M.; Meixner, A. J.; Martin, O. J. F. ACS nano 2012, 6, (11), 9828-9836.

28. Lombardi, J. R.; Birke, R. L. The Journal of Physical Chemistry C 2008, 112, (14), 5605-5617.

29. Mueller, N. S.; Heeg, S.; Reich, S. Phys. Rev. A 2016, accepted.

30. Roelli, P.; Galland, C.; Piro, N.; Kippenberg, T. J. Nature nanotechnology 2015.

31. Zhu, W.; Crozier, K. B. Nature communications 2014, 5.

32. Le, F.; Brandl, D. W.; Urzhumov, Y. A.; Wang, H.; Kundu, J.; Halas, N. J.; Aizpurua, J.; Nordlander, P. ACS nano 2008, 2, 707-718.

33. Wei, H.; Reyes-Coronado, A.; Nordlander, P.; Aizpurua, J.; Xu, H. Acs Nano 2010, 4, 2649-2654.

34. Hillenbrand, R.; Keilmann, F.; Hanarp, P.; Sutherland, D. S.; Aizpurua, J. Applied physics letters 2003, 83, 368-370.

35. Dorfmuller, J.; Dregely, D.; Esslinger, M.; Khunsin, W.; Vogelgesang, R.; Kern, K.; Giessen, H. Nano letters 2011, 11, 2819-2824.

36. Esteban, R.; Vogelgesang, R.; Dorfmuller, J.; Dmitriev, A.; Rockstuhl, C.; Etrich, C.; Kern, K. Nano letters 2008, 8, 3155-3159.

37. Kim, Z. H.; Leone, S. R. The Journal of Physical Chemistry B 2006, 110, (40), 19804-19809.

38. Rang, M.; Jones, A. C.; Zhou, F.; Li, Z.-Y.; Wiley, B. J.; Xia, Y.; Raschke, M. B. Nano letters 2008, 8, 3357-3363.

39. Olmon, R. L.; Krenz, P. M.; Jones, A. C.; Boreman, G. D.; Raschke, M. B. Optics express 2008, 16, 20295-20305.

40. Awada, C.; Plathier, J.; Dab, C.; Charra, F.; Douillard, L.; Ruediger, A. Physical Chemistry Chemical Physics 2016, 18, (14), 9405-9411.

41. Peppernick, S. J.; Joly, A. G.; Beck, K. M.; Hess, W. P. The Journal of chemical physics 2011, 134, (3), 034507-034507.

42. Batson, P. E. Ultramicroscopy 1982, 9, (3), 277-282.

43. Vesseur, E. J. R.; de Waele, R.; Kuttge, M.; Polman, A. Nano letters 2007, 7, 2843-2846.

44. Tumkur, T. U.; Yang, X.; Cerjan, B.; Halas, N. J.; Nordlander, P.; Thomann, I. Nano Letters 2016. 
45. Cang, H.; Labno, A.; Lu, C.; Yin, X.; Liu, M.; Gladden, C.; Liu, Y.; Zhang, X. Nature 2011, 469, 385388.

46. Stranahan, S. M.; Willets, K. A. Nano letters 2010, 10, 3777-3784.

47. Hubert, C.; Rumyantseva, A.; Lerondel, G.; Grand, J.; Kostcheev, S. i.; Billot, L.; Vial, A.; Bachelot, R.; Royer, P.; Chang, S.-h.; others. Nano letters 2005, 5, 615-619.

48. Lu, F.; Jin, M.; Belkin, M. A. Nature photonics 2014, 8, 307-312.

49. Stadler, J.; Oswald, B.; Schmid, T.; Zenobi, R. Journal of Raman Spectroscopy 2013, 44, (2), 227233.

50. Alonso-González, P.; Albella, P.; Schnell, M.; Chen, J.; Huth, F.; GarcYia-Etxarri, A.; Casanova, F.; Golmar, F.; Arzubiaga, L.; Hueso, L. E.; others. Nature communications 2012, 3, 684-684.

51. D’Antonio, P.; Inchingolo, A. V.; Perna, G.; Capozzi, V.; Stomeo, T.; De Vittorio, M.; Magno, G.; Grande, M.; Petruzzelli, V.; D'Orazio, A. Nanotechnology 2012, 23, (45), 455709-455709.

52. Heeg, S.; Oikonomou, A.; Fernandez-Garcia, R.; Lehmann, C.; Maier, S. A.; Vijayaraghavan, A.; Reich, S. Nano letters 2014, 14, (4), 1762-1768.

53. Ocelic, N.; Huber, A.; Hillenbrand, R. Applied Physics Letters 2006, 89, (10), 101124-101124.

54. Sivun, D.; Vidal, C.; Munkhbat, B.; Arnold, N.; Klar, T. A.; Hrelescu, C. Nano Letters 2016, 16, (11), 7203--7209.

55. Fromm, D. P.; Sundaramurthy, A.; Schuck, P. J.; Kino, G.; Moerner, W. E. Nano letters 2004, 4, (5), 957-961.

56. Hao, E.; Schatz, G. C. The Journal of chemical physics 2004, 120, (1), 357-366.

57. Maier, S. A. Optics Express 2006, 14, (5), 1957-1964.

58. Hillenbrand, R.; Keilmann, F. Applied Physics Letters 2002, 80, (1), 25-27.

59. Neuman, T.; Alonso-González, P.; Garcia-Etxarri, A.; Schnell, M.; Hillenbrand, R.; Aizpurua, J. Laser \& Photonics Reviews 2015, 9, (6), 637-649.

60. García-Etxarri, A.; Romero, I.; de Abajo, F. J. G.; Hillenbrand, R.; Aizpurua, J. Physical Review B 2009, 79, (12), 125439.

\section{Table of content graphic}

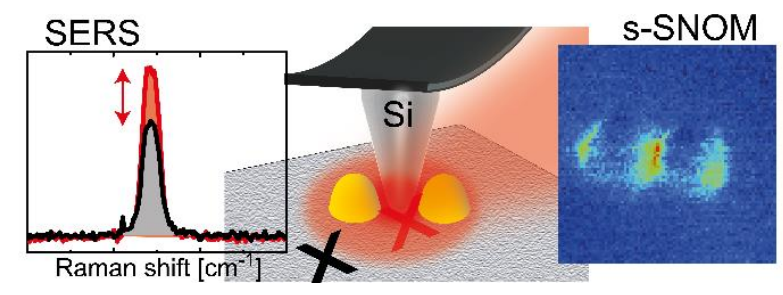

